\title{
e-Phaïstos
}

e-Phaïstos

Revue d'histoire des techniques / Journal of the history

of technology

VI-2 2017 | 2018

La diversité du patrimoine technique africain

\section{Approche topographique historique du sous-sol parisien (1800 - 2000). La ville épaisse : genèse et évolutions morphologiques}

A topographic and Historical Approach of the Parisian Underground Urban

Thickness : Creation and Morphological evolutions

\section{Mathieu Fernandez}

\section{OpenEdition}

Journals

Édition électronique

URL : http://journals.openedition.org/ephaistos/3086

DOI : 10.4000/ephaistos.3086

ISSN : 2552-0741

Éditeur

IHMC - Institut d'histoire moderne et contemporaine (UMR 8066)

Référence électronique

Mathieu Fernandez, «Approche topographique historique du sous-sol parisien (1800 - 2000). La ville épaisse : genèse et évolutions morphologiques », e-Phaïstos [En ligne], VI-2 2017 | 2018, mis en ligne le 16 novembre 2018, consulté le 09 novembre 2019. URL : http://journals.openedition.org/ephaistos/ 3086

Ce document a été généré automatiquement le 9 novembre 2019

Tous droits réservés 


\title{
Approche topographique historique du sous-sol parisien (1800 - 2000). La ville épaisse : genèse et évolutions morphologiques
}

\author{
A topographic and Historical Approach of the Parisian Underground Urban \\ Thickness : Creation and Morphological evolutions
}

Mathieu Fernandez

\author{
Thèse : références bibliographiques \\ Mathieu FERNANDEZ, Approche topographique historique du sous-sol parisien : \\ 1800-2000. La ville épaisse : genèse et évolutions morphologiques, Thèse de \\ doctorat en Histoire des techniques du CNAM, E.D. Abbé Grégoire (Paris), \\ Laboratoire HT2S, soutenue le 12 décembre 2014, un volume de texte (414 p), un \\ atlas (47 cartes), un volume d'annexes (104 p). \\ Directeur de thèse \\ André GUILLERME, Professeur, Conservatoire National des Arts et Métiers \\ Jury : \\ Sabine BARLES, Professeur, Université Paris 1 Panthéon-Sorbonne (Présidente) \\ Cedissia de CHASTENET, docteur, Architecte-voyer de la Ville de Paris \\ Youssef DIAB, professeur, Université Marne-la-Vallée \\ Maurizio GRIBAUDI, directeur d'études, EHESS \\ Éric MONIN, professeur, École Nationale d'Architecture de Paris-Val de Seine
}

En commençant, en 2011, une recherche urbaine après une formation d'ingénieur, je voulais questionner la ville, la grande ville dans la longue durée, en l'entourant à la fois de sciences humaines et de la dimension humaine des sciences abordées dans la formation d'ingénieur. Le point de départ de ma démarche a consisté à mettre en place 
des cartographies historiques à Paris au XIX ${ }^{\mathrm{e}}$ siècle. La rencontre avec le sujet du soussol parisien a été, à ce titre, une descente captivante pour mettre en convergence ces questionnements initiaux. Pour donner ici un seul exemple, l'archéologie est un domaine largement laissé de côté par l'étude technique des villes alors même qu'elle se nourrit de riches méthodologies de reconnaissance du terrain urbain.

2 L'enjeu de la thèse est de fournir un cadre, ou du moins une contribution, à un projet qui était déjà stipulé en 1933 dans le manifeste du GECUS d'Édouard Utudjian (Groupe d'Étude des Centre Urbains Souterrains), de récolement souterrain urbain pour Paris qui soit propice à alimenter à la fois des recherches en archéologie, architecture, ingénierie ou encore en reconnaissance environnementale. C'est en effet à tous ces domaines que la topographie parisienne renvoie et ceux dans lesquels la carence d'un support unifié de connaissances est sensible.

3 En histoire urbaine, les cartographies ou modèles évolutifs historiques sont plutôt rares en dehors d'expériences contenues soit par un espace restreint, soit par un phénomène unique. Partant du constat de l'absence récurrente de support de synthèse, ce qui est devenu rapidement une enquête sur la construction des disciplines et sur l'archéologie de leurs connaissances actuelles m'a conforté dans l'objectif de rendre visibles plusieurs évolutions urbaines grâce aux données historiques. J'ai donc orienté ma recherche vers ce travail de fond, qui en a dès lors occupé une part importante parallèlement au reste de la problématique exploratoire du sous-sol, lectures et projets annexes.

4 Partant de l'hypothèse de l'utilité de placer les Systèmes d'information géographique (SIG) au croisement de la problématique initiale et des ressources de l'histoire des techniques, j'ai recherché systématiquement des données anciennes brutes. L'utilité d'un SIG étant de les rapatrier sur un support actuel pour en faire des sources de première main, à la fois pour le contexte historique qui les a produites mais aussi pour l'évolution environnementale qu'elles permettent d'atteindre par diverses analyses spatiales. J'ai fait porter mes efforts sur la recherche de données dans un jeu de miroir entre celles des pionniers et leur état actuel, mais aussi sur les outils pouvant permettre leur recoupement. La recherche fut dès lors passionnante en ce sens qu'il fallait trouver un moyen de contourner ce qui avait pu être objet de limitations pour des recherches précédentes : frontières disciplinaires, techniques et épistémologiques.

Les frontières disciplinaires, j'ai tenté de les rendre poreuses après une enquête sur les origines des disciplines par le biais du domaine topographique. L'approche topographique historique du sous-sol parisien se place dans le cadre de recherches sur la longue durée exploitant les données historiques concernant plusieurs disciplines. Je me suis rendu compte après divers recoupements de sources que le premier plan de nivellement de Paris par l'ingénieur Pierre-Simon Girard de 1805 [la seule date manuscrite qu'il comporte est celle des mesures de la hauteur d'eau de la Seine le 5 prairial an XIII] est un ancêtre commun à plusieurs fils disciplinaires développés depuis. Ce plan a induit un jalon de début et un autre de fin à mon enquête sur la construction de ces savoirs urbains, histoire riche et explicative y compris sur un état actuel. D'où les bornes chronologiques de 1800 et 2000, ainsi que l'échelle des Fermiers Généraux - Paris en 1800 - : l'espace des sources les plus anciennes. Ces données ont connu un essor initial au XIX ${ }^{e}$ siècle sous l'impulsion des nouveaux champs qualifiables de pré-urbanistes. Les ingénieurs déploient le premier réseau réticulé d'adduction d'eau, suivis plus tard dans le siècle par ceux déployant le réseau des percées de voies 
nouvelles dans le tissu ancien. Dans ce cheminement, j'ai voulu accorder de l'importance à la première carte géologique de Cuviers et Brongniart en 1810, explicitement reliée au premier nivellement de la ville de Girard ou encore à la méthode de Théodore Vacquer, pionnier de l'archéologie en terrain urbain à Paris, basant sa méthode sur des outils cartographiques maitrisés jusque-là par les seuls ingénieurs. Ses manuscrits montrent toute l'importance de la représentation en relief. Nous pouvons également citer Auguste Dollot et la source désormais essentielle pour la géologie qu'il a constituée en suivant les travaux du métropolitain. Dès lors, et malgré les diverses évolutions qu'ont pu connaitre ces diverses branches d'études depuis, il m'a semblé possible de les incorporer sur un même support, comme à leur origine.

6 La frontière technique, je pense que les outils actuels peuvent permettre de la franchir sur des aspects nouveaux. Les Systèmes d'informations géographiques (SIG) sont propices au regroupement de sources diverses sur un support spatial commun intégrant les trois dimensions. Rien n'empêche d'y incorporer des données brutes, y compris altimétriques, dès lors qu'elles se représentent par un point, une ligne ou un polygone géoréférençable dans l'espace, ce qui est le cas pour les données précitées.

7 J'ai enfin recherché une solution à la frontière épistémologique, devant ce qui est un exercice difficile: tenter d'appréhender, sur une longue période et par des sources diverses des problématiques intégrées à un même espace, qu'elles soient historiques ou actuelles. J'ai cherché des réponses à ces questions qui peuvent sembler trop vastes sans structuration, en questionnant les origines et les problématiques initiales de l'urbanisme. Constituée dans les années 1920, au moment où les nouvelles écoles d'histoire et de géographie renouvèlent la conception des temporalités et surtout du rapport à l'espace, la nouvelle science spatiale de l'étude des villes a été relativement laissée à côté de ce renouvellement. La méthode de ses premiers praticiens et théoriciens se base sur les zonings dans les plans directeurs de villes : une géographie et une histoire relativement figées par une topographie cadastrale. Rarement en relief ou en épaisseur, encore plus rarement par d'autres couches que le "rez de trottoir " cadastral structurant la propriété. Ici réside une part de l'explication du manque de support commun à une échelle urbaine concernant les données des disciplines que j'ai citées précédemment. Je me suis donc inspiré d'un plan de géohistoire pour mon analyse historique de phénomènes construits sur le support SIG. Le plan de restitution de thèse choisi est justement décliné par temporalités. Il m'a fait laisser de côté un plan de type séparations disciplinaires ou bien chronologique qui aurait pu être plus instinctif, mais moins propice à explorer l'espace souterrain de manière large et à en tenter une synthèse.

8 La première partie de la thèse consiste à créer le cadre technique de travail diachronique basé sur le SIG. Plusieurs questions se posent dans une technique d'approche historique en construction environ depuis le début des années 2000. L'appréhension des sources primaire m'a poussé à comprendre l'évolution des techniques de productions cartographiques au cours des trois derniers siècles depuis le relevé de terrain jusqu'à la production de la carte telle que rencontrée dans un fonds d'archives. Cette compréhension permet d'incorporer la source brute par sa numérisation, de réaliser son géoréférencement, puis enfin de vectoriser des données à extraire. Les notions de géodésie, de points de contrôles et plus généralement de techniques de relevés anciens prennent toute leur importance lorsqu'il s'agit d'optimiser le processus de construction du SIG. La question spécifique et nouvelle de 
cette enquête était celle de la mesure du relief qui possède une histoire bien spécifique au sein de celle de la cartographie et qui n'avait jusqu'alors pas été intégrée aux premiers SIG d'analyse historique. Ce travail d'incorporation de l'archive au support numérique a permis ici de donner une application spatiale systématisée et quantitative à des travaux précédents (Barles, 1994).

9 La première partie de la thèse s'appuie sur les deux principales sources retenues renseignant le relief et la nappe phréatique, relevées au XIX ${ }^{\mathrm{e}}$ siècle (Girard, 1805, Delesse, 1858), donnant une évolution cartographiée par rapport aux données contemporaines sur l'emprise des Fermiers Généraux. Une troisième source, débutant également au XIX ${ }^{e}$ siècle, et également mise à jour depuis, est celle de l'archéologie. Les premières coupes sur l'épaisseur du substrat archéologique proviennent de Théodore Vacquer de même que le premier jet des lignes de niveaux du sol urbain originel. Nous avons utilisé la dernière mise à jour de cet héritage qui nous ait été accessible, datant de 1969, disponible sur l'Atlas Géologique de Paris, vectorisée dans le cadre du programme de recherche ANR Alpage (Krier \& Noizet, 2012). Les résultats de cette méthode systématique sur un territoire unifié donnent des cartes dépassant la chronologie initiale de l'étude. Si les sources restent des XIX et XX ${ }^{e}$ siècles, la variation du sol et des eaux de la ville sont interpolables et représentables sur toute son histoire. Des formes urbaines nouvelles apparaissent grâce aux traitements d'analyse spatiale des SIG, comme la variation de la nappe phréatique due à l'activité humaine qui commence à Paris en 1863 et dont l'apogée se situe vers 1965 ; une variation qui correspond en pratique à l'assèchement séculaire du marais parisien. Le rehaussement du sol urbain, à la temporalité millénaire et considéré en 1814 comme une solution de long terme contre les inondations de la Seine par l'ingénieur Égault, montre des formes urbaines spécifiques, épaisses, d'origine humaine. Les rabotements dus aux travaux haussmanniens deviennent également observables sur une cartographie diachronique à l'échelle de la ville.

10 La section suivante de la thèse se sert de ce matériau cartographique inscrit dans la longue durée pour interroger la phase centrale des grands travaux du XIX ${ }^{\mathrm{e}}$ siècle pour Paris, et en général la notion de projet urbain qui y fut développée. Les connaissances et les représentations disponibles permettent de saisir les ambitions et limitations des projets de ces quelques générations qui ont projeté une transformation reconnue comme phase pré-urbanistique clef. Les grandes phases que sont les topographies médicales en partie issues des travaux de la commission de salubrité de la Préfecture de la Seine au début du XIX ${ }^{e}$ siècle (Chevalier, 2012), suivies par le déploiement massif et structurant des rhizomes du réseau de l'Ourcq et des égouts dans les années 1830 et 1840 s'observent de manière cohérente et unifiée sur le modèle numérique de terrain (MNT) issu des mesures de Girard de 1805. L'échec du préfet Berger lors du percement de la seconde tranche de la rue de Rivoli suite à une erreur de nivellement ayant mené à l'explosion du budget initial et à l'arasement du quartier des Arcis dans les années qui suivirent, provoque son remplacement par le préfet Haussmann. Ce dernier transforme l'organisation interne de la préfecture de la Seine par la centralité du Service du Plan, montrant qu'une approche morphologique du pré-urbanisme est abordable par l'expérience SIG. D'un point de vue historien, plusieurs initiatives visant à la maitrise morphologique urbaine émergent dans le premier XIX ${ }^{\mathrm{e}}$ siècle : nous pouvons ici les observer. La totalisation cartographique du territoire urbain et le projet urbain de type contemporain qui en découle, s'observent dans leur construction bien avant la réorganisation signalée par les Mémoires d'Haussmann, souvent retenue comme point 
de départ de cette nouvelle façon de voir et concevoir la ville. L'enjeu est d'importance au moment où plusieurs analyses lient les transformations politique et sociale au territoire urbain (Gribaudi, 2014).

11 La dernière partie de la thèse se reconcentre sur un temps plus court, celui de la problématique plus immédiate $\mathrm{du}$ récolement urbain souterrain. Les parties précédentes ont montré qu'un tel récolement ne peut se concevoir que topohistoriquement, pour la première et principale raison que les sources sont étalées dans le temps et que leur synthèse se doit de tenir compte de cet étalement. L'impératif de gestion a rendue indispensable la mise à jour régulière du sous-sol réticulé des rues commencée par Girard, devenue carte par Emmery de Sept-Fontaines, et existant encore comme service municipal indispensable. Le sous-sol archéologique ne semble pas avoir fait l'objet de synthèse spatiale depuis plusieurs décennies à Paris en termes de couches stratigraphiques structurantes. Cependant, la cave reste le lieu où la carence d'informations est la plus importante. La dernière enquête ambitieuse date de la défense passive [Circulaires du ministère de la Guerre appliquées par les préfets de police visant à assurer la sauvegarde des populations urbaines en cas d'attaque, notamment aéroportée] dont l'analyse quantitative des archives a pu nous montrer qu'elles représentaient près de la moitié des 640000 places aménagées sous la capitale face au danger aérien durant les années 1936-1939. "L'anthropocène ", terme récent inventé dans un cadre géologique pour nommer et dater la transformation anthropique de l'écorce, trouve pourtant un espace d'étude urbain privilégié dans la cave enfouie dans des strates d'origine humaines. Le récent « souplex » la rentabilise et persiste à en faire un lieu de stockage des débordements urbains, actuellement l'absence de limitation aux prix immobiliers. Autour de la cave, l'assèchement séculaire des eaux précédemment décrit, dont le maintien artificiel par pompages et drainages est une source importante de coûts, suit chronologiquement le métabolisme décrit par André Guillerme au début du XIX ${ }^{e}$ siècle au sujet de la naissance de l'industrie, artisanale, chimique et organique, qui y a puisé une eau riche en minéraux et y a rejeté une eau usée, délétère mais riche, avant l'invention d'un réseau de drainage systématique. C'est cette même strate qui comprend le très lent processus médiéval d'accumulation qui a produit, pour ce seul exemple quantifié, la butte artificielle de Bonne-Nouvelle. Cette ancienne voirie médiévale près de la porte Saint-Denis culmine aux alentours de quinze mètres au-dessus du sol naturel.

J'en arrive aux conclusions et aux perspectives de ce travail doctoral. Je préfère, au terme de cet exposé, mettre l'accent sur la méthode de fond qui a structuré le plan de restitution et les conclusions: un ensemble de données appréhendable par des historiens pouvant faire l'objet d'une libération des données, d'un partage et d'une amélioration. Je parle ici de la technique de l'histoire au sens de l'enquête consistant à regrouper des données espacées dans le temps, à les discuter, à les transformer, à les rendre évolutives. On peut citer à ce titre Emmanuel Leroy-Ladurie lorsqu'il estime en 1967, au sujet de l'histoire du climat et des séries longues à former pour combler les carences de connaissances, que ce serait «mutiler le métier d'historien que d'en faire seulement un spécialiste en humanité ». Par ailleurs, l'usage n'est pas qu'historien. Ce sont bien des éléments de reconnaissance actuelle qui sont en jeu autour de la couche, ici urbaine, d'origine humaine. Les reconnaissances sont affaire de mémoire et les bases de données actuelles ne sont ni complètes, ni toujours accueillantes pour de nouvelles données, ni ouvertes à leurs restitutions, par exemple lorsqu'il s'agit d'informations 
morcelées entre diverses administrations. La conclusion de cette recherche postule donc deux interprétations croisées issues de la méthode.

L'apport de l'espace à l'histoire urbaine donne un cadre interprétatif utile, peut-être même davantage pour la conception passée de l'espace que pour l'espace ancien luimême. Par l'espace, des textes s'interrogent face à une source neuve, des sources se contextualisent, se critiquent, des évolutions se saisissent. L'exemple de la méthode haussmannienne, suivant dans le siècle celles des médecins puis des ingénieurs du "service exceptionnel des eaux de Paris» en est un exemple important avec un changement d'ampleur issu d'une méthode basée sur la mesure. Ce projet arase et nivelle, y compris la finesse des enquêtes urbaines du début du siècle: la distance cartographique est formellement prise dans le projet urbain.

14 L'apport de l'histoire longue à l'environnement urbain donne des résultats spatiaux actuels. La longue durée de problématiques telles que l'appréhension du relief, de la terre et des eaux pour reprendre les termes de Dante dans une controverse florentine au XIVe siècle (Dante, 1321), fournit un cadre épistémologique dans lequel des données diachroniques se croisent et se comparent. Des problématiques corolaires telles que la connaissance spatialisée sur les crues, les lieux de pollution des villes et des sols, la gestion du foncier souterrain qui ne dispose pas de dispositif cartographique de suivi, l'archéologie qui agrège difficilement à l'échelle urbaine les données de ses sondages et de ses fouilles, peuvent certainement trouver un cadre utile dans les données spatialisées et temporalisées. Crutzen (Crutzen \& Stoemer, 2000) fait débuter «l'anthropocène » aux premières mesures de la composition ancienne de l'air dans l'Antarctique fin XVIII ${ }^{e}$ siècle. Or, nous constatons à Paris un mouvement similaire dans le contexte urbain. Le XIX ${ }^{e}$ siècle voit une accumulation de mesures tout à fait nouvelles de la ville. L'observation avec le recul historique permet d'en structurer l'idée générale, alors que l'état de dispersion actuel aurait tendance à décourager. Nous retrouvons donc également à Paris l'idée que la construction de la couche humaine est observable et appréhendable d'abord dans la volonté de la mesurer, au moins autant que dans des transformations physiques.

15 Après ces deux thèmes concluant la thèse, je voudrais conclure cet exposé sur le mouvement de la libération des données qui peut être une clef d'un regroupement des mesures accumulées ces deux derniers siècles avec un état actuel, lui-même encore peu accessible. La normalisation et la démocratisation de la donnée géographique, y compris en histoire d'une part, la donnée libre produite par des recherches publiques d'autre part, peuvent être un cadre concret d'échange. Plusieurs initiatives réalisées ces dernières années ont tendance à le démontrer et j'y inscrits mes perspectives de recherches.

\section{BIBLIOGRAPHIE}

BARLES Sabine, La ville délétère. Médecins et ingénieurs dans l'espace urbain aux XVIII -XIX ${ }^{e}$ siècles, Seyssel, Champ Vallon, 1999. 
CHEVALLIER Fabienne, La naissance du Paris moderne : l'essor des politiques d'hygiène (1788-1855), Rennes, PUR, 2010.

CRUTZEN Paul J. et STOERMER Eugene F. « The Anthropocene », Global Change Newsletter 41, International Geosphere-Biosphere Program (IGBP), 2000, p. 17-18.

DANTE Alighieri, « Querelle de l'eau et de la terre », Euvres complètes, Gallimard, 1965 (éd. or. 1321).

DELESSE Achille, Carte hydrologique de la ville de Paris : 1858, publiée d'après les ordres de Mr le baron G. E. Haussmann, préfet de la Seine et exécutée par Mr Delesse, dessinée par Al. Babinski, Paris, F. Savy, 1858.

ÉGAULT Pierre, Mémoire sur les inondations de Paris, Paris, Firmin Didot, 1814.

EMMERY de SEPT-FONTAINES Henri-Charles, Statistique des eaux de la Ville de Paris (année 1839), Paris, Carilian-Goeury Dalmont, 1840.

GIRARD P.-S., VERNIQUET E., Plan de nivellement général de la ville de Paris rapporté sur l'Atlas du plan général de la ville de Paris de Verniquet, Paris, 1810.

GUILLERME André, La naissance de l'industrie à Paris. Entre sueurs et vapeurs (1770-1830), Seyssel, Champ Vallon, 2008.

GRIBAUDI Maurizio, Paris, ville ouvrière : une histoire occultée (1789-1848), Paris, La Découverte, 2014.

LEROY-LADURIE Emmanuel, Histoire du climat depuis l'an mil, Paris, Flammarion, 1967.

NOIZET Hélène, BOVE Boris, COSTA Laurent, Paris : de parcelles en pixels, Paris, Saint-Denis, P. U. de Vincennes, Comité d'histoire de la Ville de Paris, 2013.

\section{RÉSUMÉS}

Le sous-sol de Paris est reconnu et topographié depuis environ deux siècles par plusieurs disciplines. Dans cette thèse, nous avons retenu et analysé depuis leurs genèses, souvent intimement liées sur le territoire urbain, ces disciplines de l'épaisseur : la géologie, l'hydrogéologie, l'archéologie, la topographie, puis ce que nous nommons actuellement le génie urbain. L'urbanisme, accepté comme " science de l'organisation spatiale des villes ", possède peu de méthodes d'appréhension à même de synthétiser dans le temps et l'espace la diversité de ces approches. La méthode proposée par la thèse rend représentables et quantifiables plusieurs évolutions longues issues des " mesures de ville " étalées dans le temps, à travers un Système d'Information Géographique construit dans une perspective diachronique. Il concerne ici spécifiquement la verticalité. Plusieurs cartographies originales concernant le mouvement du sol et de la nappe phréatique en sont issues. Sur la base de ce support, intégrant la topographie historique, environnementale et tridimensionnelle de plusieurs disciplines urbaines, nous développons deux pistes d'analyse. La première est la compréhension de la construction du projet urbain au XIX ${ }^{\mathrm{e}}$ siècle jusqu'aux « grands travaux » à la lumière de la mesure de l'espace et des représentations disponibles. Une chronologie spécifique est proposée. La seconde est une proposition pour un récolement urbaniste souterrain appréhendant le temps long et l'épaisseur de la ville. Elle peut contribuer aux bases de données urbaines, actuellement en majorité construites sur un espace conçu comme parcellaire. Le modèle vise donc à fournir une contribution et de nouvelles pistes de recherches à la fois pour la morphologie préindustrielle de Paris, pour l'archéologie industrielle et plus généralement pour l'approche urbaniste de la 
couche, désormais connue dans un cadre géologique et environnemental, mais encore non définie du point de vue stratigraphique, sous le nom d'anthropocène.

For more than two centuries now, researchers from different backgrounds and disciplines have started studying and making topographical surveys of the Parisian underground. These different ways of studying urban thickness all inform this thesis. They are all analyzed from their very origins, which are often closely linked in an urban context. These methods include : geology, hydrology, archeology, topography, and what is currently called urban engineering. Urbanism, although it is often described as "the science of spatial organization in cities", can seldom yield as much temporal and spatial information as these various approaches can when put together. This thesis proposes to present and quantify several long-term evolutions, traced through a series of "urban measurements" performed over time by a Geographical Information System, which was designed for the purposes of a diachronic study. The system here specifically targets urban verticality. It has resulted in the creation of many original maps of ground and groundwater movement. Based on these maps, which include historical, environmental and tridimensional topography, two analyses are then conducted. The first deals with understanding the construction of an urban project in the 19th century - up until the "grands travaux" - by looking at space measurement and available representations. A specific chronology is then proposed. The second analysis offers to compile information concerning the thickness of the urban underground and its evolution through time. This could then dispense information to urban databases, many of which are currently based on an urban space fragmented by plots. The model thus built serves to contribute and to give new research perspectives, to the study of Parisian preindustrial morphology, and industrial archeology, and more generally the urban approach of the layer - known in geological and environmental contexts, but which has yet to be defined from a statigraphic point of view - under the name anthropocene.

\section{INDEX}

Mots-clés : histoire des techniques, ville, urbanisme, sous-sol, environnement, système d'information géographique (SIG)

Keywords : history of technology, town, urban planning, underground, environment, geographic information system (GIS)

Thèmes : Positions de thèse

\section{AUTEUR}

\section{MATHIEU FERNANDEZ}

Après un cursus d'ingénieur en génie urbain et une licence en histoire, ses recherches doctorales sous la direction d'André Guillerme ont porté sur le sous-sol urbain. Le principal résultat est la création de la dimension topo-historique qui faisait défaut au sol à l'aide de sources cartographiques du XIX ${ }^{\mathrm{e}}$ siècle, remises à jour pour constituer des données compatibles avec les systèmes d'information géographiques (SIG). Il réalise actuellement un post-doctorat centré sur l'adaptation des méthodes du métabolisme urbain (reconnaissance des flux et stocks de matière mobilisés par les activités anthropiques d'un territoire) aux projets d'urbanisme mis en œuvre par le projet politique du Grand Paris (réseaux et constructions neuves). 